\title{
A SCHEDULING ALGORITHM FOR REDUCING UNUSED TIMESLOTS BY CONSIDERING HEAD GAP AND TAIL GAP IN TIME SLICED OPTICAL BURST SWITCHED NETWORKS
}

Takanori Ito, Daisuke Ishii, Kohei Okazaki, Naoaki Yamanaka, and Iwao Sasase Dept. of Information and Computer Science, Keio University

Yokohama, Kanagawa, Japan 223-8522

Abstract: We propose a scheduling algorithm for reducing unused timeslots by considering head gap and tail gap newly generated by assigning a data burst in order to improve the burst loss probability and the throughput performances in Time Sliced Optical Burst Switched (TSOBS) networks. The proposed scheduling algorithm selects the timeslot in which either head gap or tail gap newly generated becomes the minimum. We show that the proposed scheduling algorithm can improve the burst loss probability and the throughput performances as compared with the conventional one.

\section{INTRODUCTION}

Optical Burst Switching (OBS) has been proposed as a new scheme to realize IP over WDM networks [1]- [4]. In OBS networks, at a core router, when two or more data bursts arrive at the same output port simultaneously, the burst contention occurs. When the burst contention occurs, either burst is discarded. In order to avoid the burst loss due to the burst contention, several scheduling algorithms using a wavelength converter and an optical buffer implemented through a bundle of fiber delay lines (FDLs) have been proposed [1], [2]. The wavelength converters using $\mathrm{O} / \mathrm{E}$ and $\mathrm{E} / \mathrm{O}$ conversions increase the electric processing at core routers. Although the all-optical wavelength converters without $\mathrm{O} / \mathrm{E}$ and $\mathrm{E} / \mathrm{O}$ conversions are desirable and have been developed over a number of years, all-optical wavelength converters are not still in practical use because of the issues of the performance and cost implications. On the other hand, although FDLs can be implemented, using a large number of FDLs causes an increase of optical hardware volume and noise level due to 
the transmission of optical signals in FDLs. In OBS networks, since a burst length is long, the buffer size of FDLs needed in core routers becomes large. Therefore, there is the problem that optical hardware volume and noise level increase in OBS networks.

Time Sliced Optical Burst Switching (TSOBS) [3] has been proposed as an OBS system which does not use wavelength converters and can reduce the buffer size of FDLs needed in core routers. In TSOBS networks, the wavelength used for transmission of data bursts is divided into frames, and each frame is subdivided into several timeslots. In edge routers, a generated data burst is divided into the several data of one timeslot length and the divided data burst is transmitted in the same timeslot every frame. TSOBS can avoid the burst contention by using short FDLs in core routers since the data transmitted in the network are short. Therefore, TSOBS can reduce the buffer size of FDLs needed in core routers as compared with OBS. In TSOBS networks, when a certain timeslot in each frame is focused on, the empty timeslots may exist between two different data bursts already assigned to the timeslot. The group of the empty timeslots is called gap in this paper. The value of gap is defined as the number of the empty timeslots. In a core router, the data burst can be transmitted by using the gap if there is the gap which is longer than the length of the data burst. And, we call the timeslot, in which the data burst can be transmitted, the available timeslot. However, in TSOBS networks, a data burst is assigned to the available timeslot which is found first without considering gaps newly generated by assigning the data burst, and hence, a large number of small gaps where only a short burst can be assigned are generated. Therefore, the conventional scheduling algorithm increases the possibility that a long burst will be discarded. As a result, it causes the degradation of the burst loss probability and the throughput performances.

In this paper, in order to improve the burst loss probability and the throughput performances, we propose a scheduling algorithm for reducing unused timeslots by considering head gap and tail gap newly generated by assigning a data burst. The proposed scheduling algorithm selects the timeslot in which either head gap or tail gap newly generated becomes the minimum. The proposed scheduling algorithm can increase the possibility that a long burst will be assigned, since longer gaps can be generated in other timeslots by assigning the data burst to the timeslot in which the gap newly generated becomes the minimum. Therefore, the proposed scheduling algorithm can improve the burst loss probability and reduce unused timeslots on data wavelengths. We compare the performances of the proposed scheduling algorithm with that of the conventional one with respect to the burst loss probability and the throughput by computer simulations. As a result, we show that the proposed scheduling algorithm can improve the burst loss probability and the throughput performances as compared with the conventional one. 


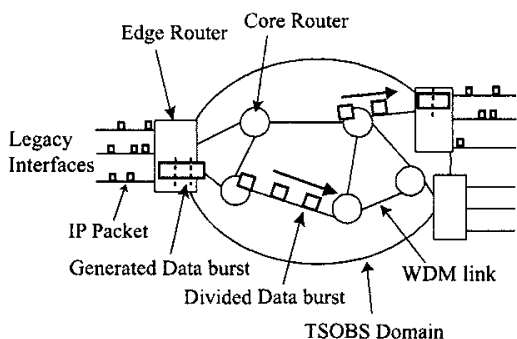

Figure 1. A TSOBS network.

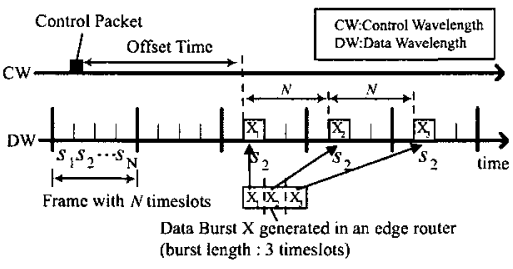

Figure 2. An example of burst transmission at an edge router.

\section{TSOBS NETWORKS}

Fig. 1 shows a TSOBS network. Edge routers assemble several IP packets with the same destination into a burst. And edge routers divide a generated data burst into the several data of one timeslot length and transmit the divided data burst every fixed interval. Core routers carry out the scheduling and switching of data bursts. In core routers, the divided data burst is transmitted to the next router with the divided form held. Fig. 2 shows an example of burst transmission at an edge router. In TSOBS networks, the wavelength used for transmission of data bursts is divided into frames, and each frame is subdivided into $N$ timeslots. Edge routers first transmit a control packet to reserve an output timeslot in a core router before a data burst is transmitted. Subsequently, edge routers transmit a data burst on a separate wavelength after some offset time. The offset time should also allow core routers at each hop along the path to have enough time to process the control packet before its corresponding data burst arrives. In this paper, the offset time would be proportional to the number of hops which the burst will traverse. Therefore, the offset time differs in each burst. Each control packet includes the address information, the wavelength and timeslot in which the data burst is transmitted, the offset time which identifies the frame in which the first data of the divided data burst is transmitted, and the burst length which identifies the number of timeslots used to transmit the data burst. As shown in Fig. 2, in an edge router, a generated data burst is divided into the several data of one timeslot length and is transmitted in the same timeslot every frame. In core routers, the switching of data bursts is done entirely in the optical domain. Core routers dynamically switch a divided data burst from an incoming timeslot to an output timeslot on the appropriate outgoing link. In core routers, when the burst contention occurs, the output timeslot of the data burst is changed by using FDLs in order to avoid the burst contention. The unit delay time of FDLs is one timeslot and the maximum delay which can be given to the data bursts by FDLs is $N$ timeslots.

In the conventional scheduling algorithm, core routers search the statuses 


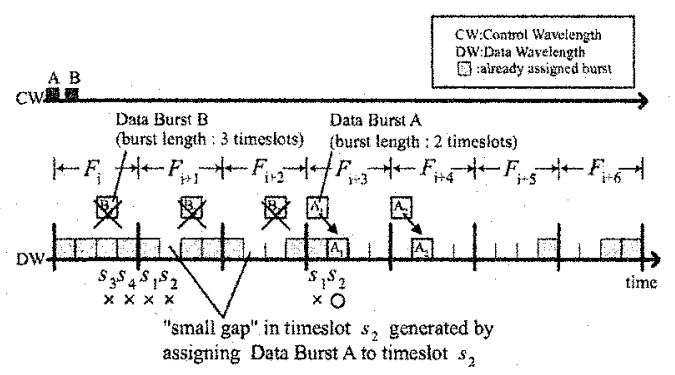

Figure 3. An example of the conventional scheduling algorithm $(N=4)$.

of the timeslots in sequence from the timeslot in which a data burst will arrive, and the data burst is assigned to the available timeslot which is found first [3]. Core routers can search the statuses of $N$ timeslots at the maximum in sequence from the timeslot in which a data burst will arrive. If all the timeslots are unavailable, the data burst is discarded. Fig. 3 shows an example of the conventional scheduling algorithm $(N=4)$. The control packets of data burst A and B arrive at a core router in order, as shown in Fig. 3. First, the core router analyzes the control packet of data burst $A$, and obtains the information that the burst length of data burst $\mathrm{A}$ is 2 timeslots and data burst $\mathrm{A}$ arrives in timeslot $s_{1}$ of frame $F_{i+3}$. Next, the core router searches the statuses of the timeslots in sequence from timeslot $s_{1}$ in which data burst A will arrive. Since other data burst is already assigned to timeslot $s_{1}$ in $F_{i+3}$, the core router searches the status of timeslot $s_{2}$. Timeslot $s_{2}$ is empty in two consecutive frames of $F_{i+3}$ and $F_{i+4}$. Therefore, data burst $\mathrm{A}$ is assigned to timeslot $s_{2}$. Then, the core router analyzes the control packet of data burst $\mathrm{B}$, and obtains the information that the burst length of data burst B is 3 timeslots and data burst $B$ arrives in timeslot $s_{3}$ of frame $F_{i}$. Timeslot $s_{3}, s_{4}, s_{1}$, and $s_{2}$ are not empty in three consecutive frames. Therefore, data burst $B$ is discarded. In the conventional scheduling algorithm, a data burst is assigned to the available timeslot which is found first without considering gaps newly generated by assigning the data burst, and hence, a large number of small gaps where only a short burst can be assigned are generated. Therefore, the conventional scheduling algorithm increases the possibility that a long burst will be discarded. As a result, it causes the degradation of the burst loss probability and the throughput performances.

\section{PROPOSED SCHEDULING ALGORITHM}

In order to improve the burst loss probability and the throughput performances, we propose a scheduling algorithm for reducing unused timeslots by considering head gap and tail gap newly generated by assigning a data burst. The proposed scheduling algorithm selects the timeslot in which either head gap or tail gap newly generated becomes the minimum. The proposed scheduling algorithm can increase the possibility that a long burst will be assigned, since longer gaps can be generated in other timeslots by assigning the data 

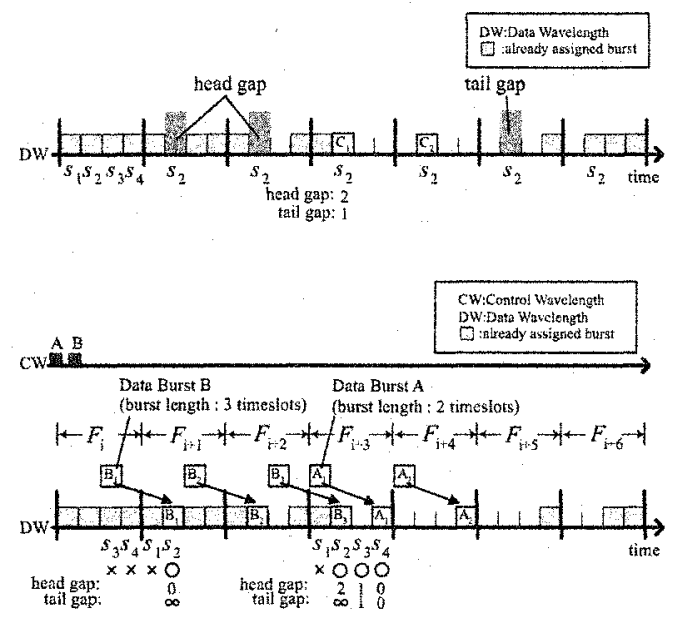

Figure 4. An example of head gap and tail gap $(N=4)$

Figure 5. An example of the proposed scheduling algorithm $(N=4)$.

burst to the timeslot in which the gap newly generated becomes the minimum. Therefore, the proposed scheduling algorithm can improve the burst loss probability and reduce unused timeslots on data wavelengths. Fig. 4 shows an example of head gap and tail gap $(N=4)$. When data burst $\mathrm{C}$ is assigned to timeslot $s_{2}$, the head gap is 2 since timeslot $s_{2}$ is empty in two consecutive frames which exist ahead of data burst $\mathrm{C}$, and the tail gap is 1 since timeslot $s_{2}$ is empty in one frame which exists behind data burst $\mathrm{C}$.

Here, we explain the flow of the proposed scheduling algorithm. First, core routers search the statuses of $N$ timeslots in sequence from the timeslot in which a data burst will arrive. Simultaneously, if the searched timeslot is available, core routers compute head gap and tail gap newly generated by assigning the data burst to the timeslot. After core routers search the statuses of all the timeslots, core routers compare head gap and tail gap in all the available timeslots and select the timeslot in which either head gap or tail gap becomes the minimum. When there are two or more timeslots in which a gap becomes the minimum, the proposed scheduling algorithm selects the timeslot in which tail gap becomes the minimum in order to use gaps as early as possible. Furthermore, when there are two or more candidate timeslots, the proposed scheduling algorithm selects the timeslot in which the delay given to the data burst by FDLs is the minimum in order not to increase the delay of data bursts. Fig. 5 shows an example of the proposed scheduling algorithm $(N=4)$. In Fig. 5 , the control packets of data burst A and B arrive at a core router in order, like Fig. 3. First, the core router analyzes the control packet of data burst A, and obtains the information that the burst length of data burst $A$ is 2 timeslots and data burst $\mathrm{A}$ arrives in timeslot $s_{1}$ of frame $F_{i+3}$. Next, the core router searches the statuses of all the timeslots in sequence from timeslot $s_{1}$ in which data burst A will arrive. Simultaneously, the core router computes head gap 
and tail gap in timeslot $s_{2}, s_{3}$, and $s_{4}$, since timeslot $s_{2}, s_{3}$, and $s_{4}$ are the available timeslots. In Fig. 5, when data burst $\mathrm{A}$ is assigned to timeslot $s_{4}$, both head gap and tail gap newly generated become the minimum. Therefore, data burst $\mathrm{A}$ is assigned to timeslot $s_{4}$. Then, the core router analyzes the control packet of data burst $B$, and obtains the information that the burst length of data burst $\mathrm{B}$ is 3 timeslots and data burst B arrives in timeslot $s_{3}$ of frame $F_{i}$. Similarly, the core router carries out the scheduling of data burst B. And data burst $\mathrm{B}$ is assigned to timeslot $s_{2}$. In the conventional scheduling algorithm, data burst B is discarded since a small gap is generated in timeslot $s_{2}$ by assigning data burst A to timeslot $s_{2}$, as shown in Fig. 3. On the other hand, in the proposed scheduling algorithm, data burst B can be assigned to timeslot $s_{2}$ since the longer gap can be generated in timeslot $s_{2}$ by assigning data burst A to timeslot $s_{4}$, as shown in Fig. 5. Therefore, the proposed scheduling algorithm can reduce unused timeslots by selecting the timeslot in which either head gap or tail gap newly generated becomes the minimum.

\section{PERFORMANCE EVALUATION}

In this section, we compare the burst loss probability and the throughput of the proposed scheduling algorithm with those of the conventional one by computer simulations. The throughput is defined as the ratio of the amount of data transmitted per unit time to the transmission rate. We assume that the switch size of a core router is 16 , the transmission rate on each wavelength is $40 \mathrm{Gbps}$, frames are subdivided into $N$ timeslots and the length of timeslot is $1 \mu \mathrm{s}$. The input has uniform traffic with rate $\rho$. And, all bursts arriving at each input port of a core router have variable length bursts. The burst length has the uniform distribution of 1 to 5 timeslots length. The number of hops to a destination edge router has the uniform distribution of 1 to 16 hops. The processing time of a control packet in each core router is $10 \mu \mathrm{s}$ [4].

Fig. 6 shows the burst loss probability versus input load $\rho$. From Fig. 6, we show that the proposed scheduling algorithm can reduce the burst loss probability as compared with the conventional one. The reason is as follows. In the conventional scheduling algorithm, a data burst is assigned to the available timeslot which is found first without considering gaps newly generated by assigning the data burst, and hence, a large number of small gaps where only a short burst can be assigned are generated. Therefore, the conventional scheduling algorithm increases the possibility that a long burst will be discarded and the number of data bursts which can be assigned decreases. On the other hand, in the proposed scheduling algorithm, a data burst is assigned to the timeslot in which either head gap or tail gap newly generated by assigning the data burst becomes the minimum. The proposed scheduling algorithm can increase the possibility that a long burst will be assigned since longer gaps can be generated 


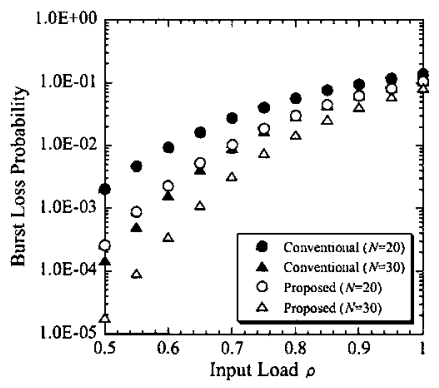

Figure 6. The burst loss probability versus input load $\rho$.

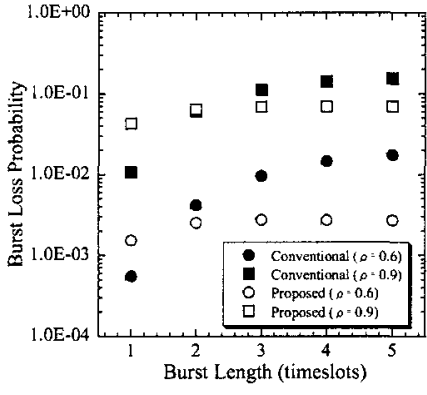

Figure $7 . \quad$ The burst loss probability by the burst length $(N=20)$.

in other timeslots by assigning the data burst to the timeslot in which the gap newly generated becomes the minimum. Therefore, the number of data bursts which can be assigned increases. Then, in order to prove the reason mentioned above, we show the burst loss probability by the burst length in Fig. 7. From Fig. 7, we find that the conventional scheduling algorithm degrades the loss probability of a long burst as compared with that of a short burst. This is because, the conventional scheduling algorithm increases the possibility that a short burst will be assigned as compared with the possibility that a long burst will be assigned since a large number of small gaps where only a short burst can be assigned are generated. On the other hand, we find that the proposed scheduling algorithm reduces the loss probability of a long burst. This is because, the proposed scheduling algorithm can assign a short burst and a long burst with the almost same possibility since the number of small gaps where only a short burst can be assigned decreases and longer gaps can be generated. From Figs. 6 and 7, we show that the proposed scheduling algorithm can increase the number of data bursts which can be assigned since the proposed scheduling algorithm can reduce the loss probability of a long burst. And as shown in Fig. 7, in the conventional scheduling algorithm, the burst loss probability is affected by the burst length and a long burst is more likely to be discarded than a short burst. However, in the proposed scheduling algorithm, the burst loss probability does not depend on so much the burst length and the difference of the burst loss probabilities by the burst length can be small.

Fig. 8 shows the throughput performance versus input load $\rho$. From Fig. 8, we show that the proposed scheduling algorithm can improve the throughput performance as compared with the conventional one. The reason is as follows. In the conventional scheduling algorithm, since the scheduling is carried out without considering gaps newly generated, a large number of small gaps where only a short burst can be assigned are generated and the number of data bursts which can be assigned decreases. Therefore, the number of unused timeslots increases and the amount of data which can be transmitted per unit time de- 


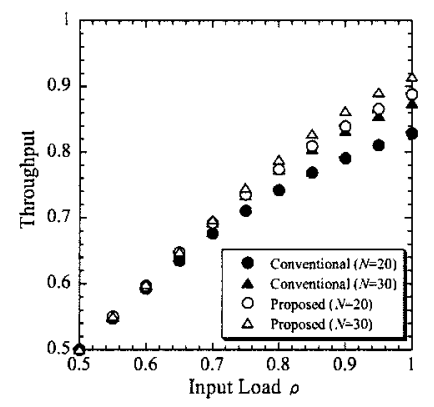

Figure 8. The throughput performance versus input load $\rho$.

creases. On the other hand, in the proposed scheduling algorithm, since the scheduling is carried out with considering gaps newly generated, longer gaps can be generated and the number of data bursts which can be assigned increases. Therefore, the number of unused timeslots can be reduced and the amount of data which can be transmitted per unit time increases.

\section{CONCLUSION}

We have proposed a scheduling algorithm for reducing unused timeslots by considering head gap and tail gap newly generated by assigning a data burst in order to improve the burst loss probability and the throughput performances in TSOBS networks. The proposed scheduling algorithm selects the timeslot in which either head gap or tail gap newly generated becomes the minimum. By computer simulations, we find that the proposed scheduling algorithm can improve the burst loss probability and the throughput performances as compared with the conventional one.

\section{ACKNOWLEDGMENT}

This work is partly supported by Keio University The 21 st Century COE Program on "Optical and Electronic Device Technology for Access Network" and Japan Society for the Promotion of Science(JSPS).

\section{REFERENCES}

[1] C. Qiao and M. Yoo, "Optical burst switching(OBS)-a new paradigm for an optical internet," J.High Speed Networks, vol.8, no.1, pp.69-84, Jan. 1999.

[2] Y. Xiong, M. Vandehoute, and H. C. Cankaya, "Control architecture in optical burst-switched WDM networks," IEEE J. Select. Areas Commun., vol.18, no.10, pp.1838-1851, Oct. 2000.

[3] J. Ramamirtham and J. Turner, "Time sliced optical burst switching," Proc. INFOCOM 2003, vol.3, pp.2030-2038, San Francisco, U.S.A., Apr. 2003.

[4] B. C. Kim, Y. Z. Cho, J. H. Lee, Y. S. Choi, and D. Montgomery, "Performance of optical burst switching techniques in multi-hop networks," Proc. GLOBECOM 2002, vol.3, pp.2772-2776, Taipei, Taiwan, Nov. 2002. 